\title{
Evidence for Eccentric, Precessing Gaseous Debris in the Circumstellar Absorption toward WD $1145+017$
}

\author{
P. Wilson Cauley ${ }^{1,2}$, Jay Farihi ${ }^{3}$, Seth Redfield ${ }^{1}$ (D), Stephanie Bachman ${ }^{1,4}$, Steven G. Parsons ${ }^{5}$, and Boris T. Gänsicke ${ }^{6}$ \\ ${ }^{1}$ Astronomy Department, Wesleyan University, Van Vleck Observatory, Middletown, CT, USA; pwcauley@gmail.com \\ ${ }^{2}$ School of Earth and Space Exploration, Arizona State University, Tempe, AZ, USA \\ Department of Physics and Astronomy, University College London, London, UK \\ ${ }_{5}^{4}$ Department of Physics, Indiana University of Pennsylvania, Indiana, PA, USA \\ ${ }^{5}$ Department of Physics and Astronomy, University of Sheffield, Sheffield, UK \\ ${ }^{6}$ Department of Physics, University of Warwick, Coventry, UK \\ Received 2017 November 9; revised 2017 December 21; accepted 2017 December 21; published 2018 January 5
}

\begin{abstract}
We present time-series spectra revealing changes in the circumstellar line profiles for the white dwarf WD $1145+017$. Over the course of 2.2 years, the spectra show complete velocity reversals in the circumstellar absorption, moving from strongly redshifted in 2015 April to strongly blueshifted in 2017 June. The depth of the absorption also varies, increasing by a factor of two over the same period. The dramatic changes in the line profiles are consistent with eccentric circumstellar gas rings undergoing general relativistic precession. As the argument of periapsis of the rings changes relative to the line of sight, the transiting gas shifts from receding in 2016 to approaching in 2017. Based on the precession timescales in the favored model, we make predictions for the line profiles over the next few years. Spectroscopic monitoring of WD $1145+017$ will test these projections and aid in developing more accurate white dwarf accretion disk models.
\end{abstract}

Key words: circumstellar matter - techniques: spectroscopic - white dwarfs

\section{Introduction}

The polluted atmospheres observed in at least $\approx 30 \%$ of white dwarfs are caused by the accretion of planetesimal debris, the result of tidal disruption of rocky bodies by the host star, and the eventual collisional grinding of the debris into dust (Zuckerman et al. 2010; Koester et al. 2014b; Kenyon \& Bromley 2017b). In some systems, the disrupted planetesimal material is detected in disks of dust and gas (see Farihi 2016, and references therein). The atmospheric abundances of heavy elements reflects the composition of the orbiting and infalling planetesimal debris, and hence the bulk composition of the disrupted parent body. Polluted white dwarf atmospheres thus offer an exciting window into the chemistry and assembly of the planets and minor bodies formed in the system (Gänsicke et al. 2012; Farihi et al. 2013; Xu et al. 2013; Jura \& Young 2014; Raddi et al. 2015).

WD $1145+017$ was the first system for which transits associated with closely orbiting planetesimals were detected: Vanderburg et al. (2015) presented K2 light curves consistent with large clouds of opaque debris orbiting near the stellar Roche limit. Subsequent photometric monitoring has revealed significant evolution of the transit depths and morphologies (Gänsicke et al. 2016; Rappaport et al. 2016; Croll et al. 2017). $\mathrm{Xu}$ et al. (2016) presented high-resolution spectra of WD $1145+017$ that showed circumstellar gas lines were present in several metal species with depths $\approx 10 \%-30 \%$ below the continuum. The redshifted line components exhibited maximum velocities of $\approx 200 \mathrm{~km} \mathrm{~s}^{-1}$, and significant absorption extending down to $\approx 0 \mathrm{~km} \mathrm{~s}^{-1}$, indicative of material receding along the line of sight across a wide range of velocities. Redfield et al. (2017) presented multi-epoch spectra that displayed short- and long-term variability of the circumstellar absorption, and suggested an eccentric circumstellar disk model to account for the redshifted velocities.
In this paper, we present a multi-year monitoring campaign of the circumstellar absorption toward WD $1145+017$. The data reveal that the absorption velocities undergo a gradual change from strongly redshifted to strongly blueshifted over the course of 2.2 years. The change from redshifted to blueshifted velocities is evidence that the absorbing gas is not infalling onto the star. We model the line profiles using eccentric rings that are precessing on a timescale similar to what is predicted by general relativity for the mean periastra of the rings, shifting the line-of-sight absorbing material from moving away from us to toward us with a precession period of $\approx 5.3$ years. We derive circumstellar line profile predictions based on the ring model to be tested with future spectroscopic monitoring. In Section 2, we briefly describe the observations and data reduction steps. Section 3 details the eccentric ring model and Section 4 is a discussion of the model and other possible explanations for the velocity changes in the line profiles. Finally, a brief summary and our conclusions are give in Section 5.

\section{Observations and Data Reduction}

The data were obtained using two different instruments: HIRES on Keck I (Vogt et al. 1994) and X-shooter on the VLT (Vernet et al. 2011). The dates and details of the observations are given in Table 1. Further information concerning these data can be found in Xu et al. (2016) and Redfield et al. (2017), respectively.

The 2016 and 2017 Keck data sets were collected using the same instrument settings as the other Keck observations. The reduced 2015 April 11 data was previously published (Xu et al. 2016). All 2016 and 2017 Keck data were reduced using the HIRES Redux package ${ }^{7}$ following typical reduction steps. The 2017 February $14 \mathrm{X}$-shooter data were obtained with the same

\footnotetext{
7 Written by Jason X. Prochaska; http://www.ucolick.org/ xavier/ HIRedux/index.html.
} 
Table 1

Observations

\begin{tabular}{|c|c|c|c|c|c|c|c|c|}
\hline $\begin{array}{l}\text { Date } \\
\text { (UT) } \\
\text { (1) }\end{array}$ & $\begin{array}{l}\text { Instrument } \\
\text { (2) }\end{array}$ & $\begin{array}{c}\text { Program ID } \\
\text { (3) }\end{array}$ & $\begin{array}{l}N_{\exp } \\
(4)\end{array}$ & $\begin{array}{l}t_{\exp }^{a} \\
(s) \\
(5)\end{array}$ & $\begin{array}{c}\mathrm{S} / \mathrm{N} \\
\left(\begin{array}{c}5000 \AA) \\
(6)\end{array}\right.\end{array}$ & $\begin{array}{c}\text { Slit Width }{ }^{\mathrm{a}} \\
\text { (7) }\end{array}$ & $\begin{array}{c}\lambda / \Delta \lambda \\
(8)\end{array}$ & $\begin{array}{c}\text { Spectral Range } \\
\text { (§̊) } \\
\text { (9) }\end{array}$ \\
\hline 2015 Apr 11 & HIRES & $\mathrm{U} 027 \mathrm{Hb}$ & 3 & 2400 & 25 & 1."148 & 37,000 & $3100-5950$ \\
\hline 2015 Nov 14 & HIRES & $\mathrm{N} 116 \mathrm{Hb}$ & 2 & 1200 & 10 & 1."148 & 37,000 & $3100-5950$ \\
\hline 2016 Feb 14 & X-shooter & 296.C-5014(A) & 40 & 300 & 120 & $0 . " 9$ & 7000 & $3000-10,000$ \\
\hline 2016 Mar 29 & $\mathrm{X}$-shooter & 296.C-5014(D) & 29 & 280 & 130 & $0 . " 9$ & 7000 & $3000-10,000$ \\
\hline 2016 Apr 8 & $\mathrm{X}$-shooter & 296.C-5014(B) & 44 & 280 & 140 & $0 . " 9$ & 7000 & $3000-10,000$ \\
\hline 2016 Jun 3 & HIRES & $\mathrm{N} 171 \mathrm{Hb}$ & 3 & 1200 & 13 & 1."148 & 37,000 & $3500-7400$ \\
\hline 2017 Feb 14 & X-shooter & 598.C-0695(A) & 48 & 280 & 120 & $0 . " 9$ & 7000 & $3000-10,000$ \\
\hline 2017 May 17 & HIRES & $\mathrm{N} 188 \mathrm{Hb}$ & 3 & 1800 & 16 & 1."148 & 37,000 & $3500-7400$ \\
\hline 2017 Jun 26 & HIRES & $\mathrm{N} 188 \mathrm{Hb}$ & 3 & 1200 & 18 & 1."148 & 37,000 & $3500-7400$ \\
\hline
\end{tabular}

Note.

${ }^{\mathrm{a}} \mathrm{X}$-shooter exposure times and slit widths are listed for the UVB arm.

settings as the other $\mathrm{X}$-shooter data and reduced in an identical manner.

Photospheric models are employed to remove the narrow atmospheric metal lines (see Koester et al. 2014a; Redfield et al. 2017, for details). The photospheric absorption is present at a velocity of $\approx 42 \mathrm{~km} \mathrm{~s}^{-1}$. We assume a mass of $0.6 M_{\text {Sun }}$, which produces a gravitational redshift of $30 \mathrm{~km} \mathrm{~s}^{-1}$ and thus a system radial velocity of $12 \mathrm{~km} \mathrm{~s}^{-1}$. Due to the large widths and maximum absorption velocities of the circumstellar line profiles, choosing a different value of the gravitational redshift does not change the interpretation of the line profiles in a meaningful way. Once the photospheric model is subtracted from the spectrum, the resulting circumstellar profile is shifted into the observer's reference frame. We note that no detectable variability is present in the photospheric metal lines.

The reduced circumstellar line profiles for Ni II $3576.764 \AA$, Mg I 5183.604 А, and Fe II 5316.615 ̊ are shown in Figure 1. All Keck profiles are binned to the X-shooter resolution. Due to the $\approx 150-200 \mathrm{~km} \mathrm{~s}^{-1}$ velocity widths of the circumstellar absorption, little information is lost in the binning. All three lines show similar evolution in time, moving from absorption with maximum redshifted velocities of $\approx 200 \mathrm{~km} \mathrm{~s}^{-1}$ in 2015 to maximum blueshifted velocities of $\approx 200 \mathrm{~km} \mathrm{~s}^{-1}$ in 2017 . This suggests that all three metal ions trace coincident regions of the circumstellar material. In the next section, we present a model that accounts for the absorption via eccentric gas rings that are precessing due to general relativistic effects of the white dwarf's gravity well.

\section{Precessing Eccentric Rings}

We model the absorption using 14 confocal, eccentric gas rings in coplanar orbits oriented edge-on to the line of sight. The rings are parameterized according to their periastra, eccentricity, and the position angle of the apsidal line measured clockwise from the line-of-sight direction (see Appendix D of Metzger et al. 2012), i.e., the apsidal lines of the rings are misaligned. It is also assumed that the rings orbit at their Keplerian velocities, ignoring pressure support that slightly reduces gas velocity (e.g., Rafikov 2011). Each ring has a radial width of $0.5 R_{*}$ and a constant azimuthal density. A density is assigned to the innermost ring, which has periastron distance $r_{\text {in }}$, for each epoch and then densities in subsequent rings orbiting at distance $r$ are scaled by a factor of $\left(r_{\text {in }} / r\right)^{2}$. The ring width is arbitrary and is degenerate with the ring density, which combine to give the optical depth through the gas. The eccentricity of the rings changes linearly from 0.25 to 0.30 for periastron distances of $18.8 R_{*}$ to $27.9 R_{*}$ and the apsidal line angle steps linearly from $205^{\circ}$ for the innermost ring to $283^{\circ}$ for the outermost ring. The ring eccentricities and apsidal line angles are shown as a function of $r_{\text {in }}$ in Figure 2. The precession period of the rings is 5.3 years, which is chosen in order to match the observed profile evolution. This is very similar to the mean precession period of 6.3 years given by general relativity for the periastron ring distances in our model, although we note that general relativistic precession is a strong function of periastron distance (Veras et al. 2014; Manser et al. 2016).

The line profiles are generated using Doppler-broadened delta functions. We take the Doppler width of the line profile to be the thermal line width at that point in the ring. For the case of Fe II, the thermal line widths are $\approx 2 \mathrm{~km} \mathrm{~s}^{-1}$ at gas temperatures of $\approx 6000 \mathrm{~K}$, which is typical for the orbital distances of the ring material (Melis et al. 2010). The scale height, which determines the density as a function of vertical distance from the ring midplane, at each ring position is calculated using the same temperature approximation. The stellar surface is divided into a $100 \times 100$ Cartesian grid the and intensity from each stellar grid point is then extincted by any obscuring ring material. We do not consider multiple scatterings through the rings. The entire stellar grid is then summed to produced the final line profile. Limb darkening is accounted for using a four-parameter Claret coefficient law for $T_{\text {eff }}=16,000 \mathrm{~K}$ and $\log g=8.0$ (Gianninas et al. 2013).

It is important to note that the ring configuration described here is almost certainly not unique in being able to broadly reproduce the observed line profiles. Furthermore, the WD $1145+017$ system is highly dynamic and new material appears to be constantly replenished through the disk. However, there are several critical constraints on the eccentric ring model. First, the combination of eccentricity, viewing angle, and periastron distance must combine to produce orbiting gas at velocities of $\pm 200 \mathrm{~km} \mathrm{~s}^{-1}$ along the line of sight. The periastra of the rings must also have general relativistic precession periods similar to that observed. Values of the eccentricity $<0.1$ cannot produce sufficient line-of-sight velocities unless the orbital distances are $\lesssim 10 R_{*}$. However, a ring with this smaller periastron distance results in a precession period of days to months, shorter than that suggested by the changing line profiles. Alternatively, periastra $\gtrsim 50 R_{*}$ require 


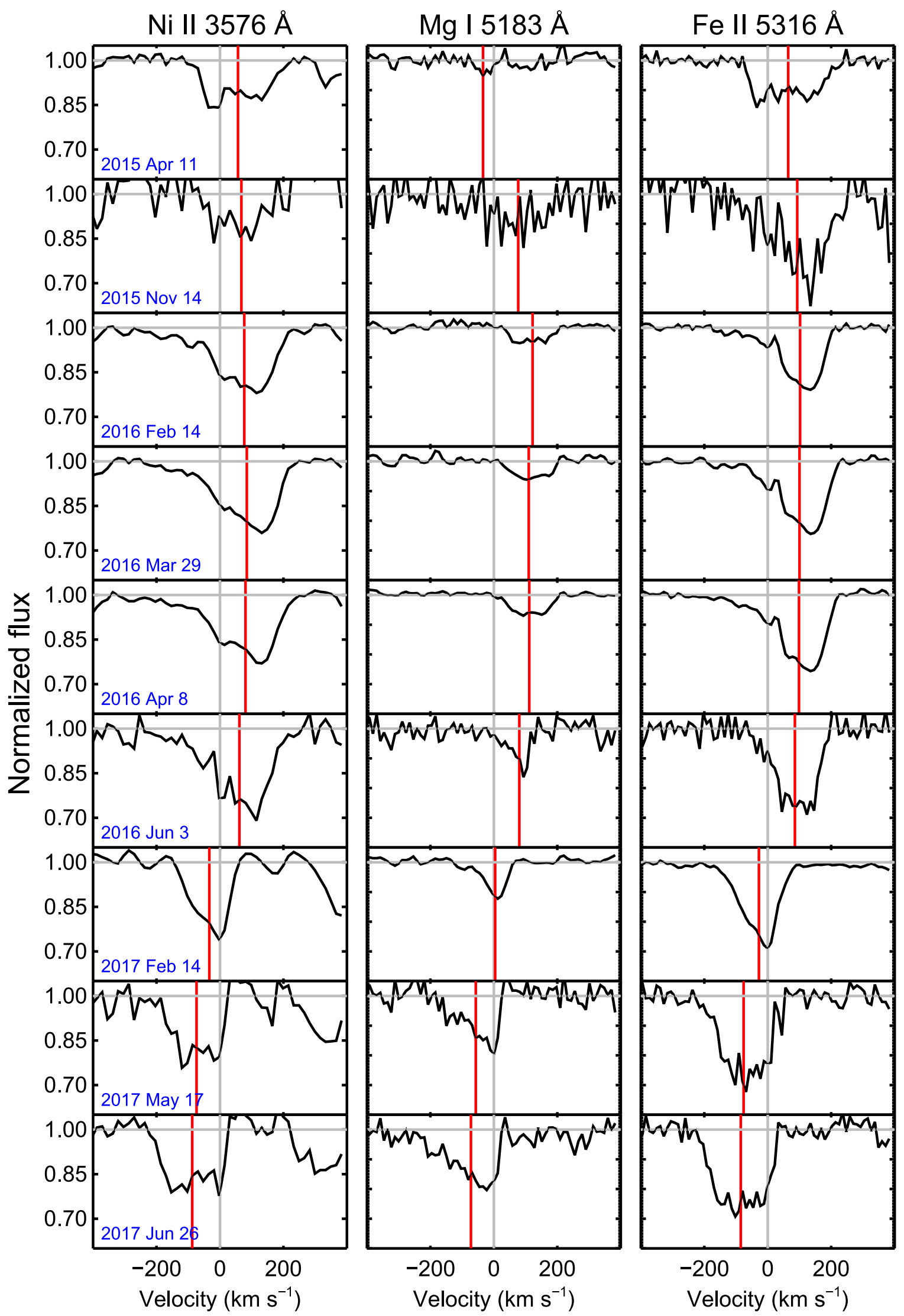

Figure 1. Circumstellar line profiles for the Ni II $3576 \AA$, Mg I $5183 \AA$, and Fe II $5316 \AA$ lines. All profiles are binned to the resolution of the X-shooter data. The normalized flux continuum and zero velocity are marked with horizontal and vertical gray lines, respectively. The photospheric absorption lines have been subtracted and all profiles are shown in the rest frame of the observer. The vertical red lines show the absorption-weighted velocity of the circumstellar line. The lines show a common evolution in terms of strength and morphology. Note the appearance of the longer wavelength lines in the Ni II profiles when the absorption changes from redshifted to blueshifted. 


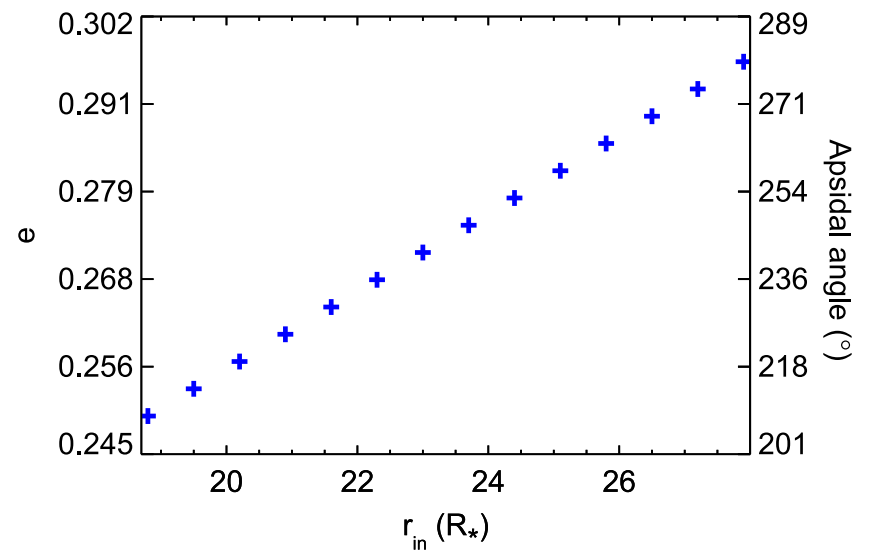

Figure 2. Periastron vs. eccentricity and apsidal angle. The apsidal line angles of the rings are misaligned and the rings become more eccentric with larger $r_{\text {in }}$.

eccentricities $>0.4$ to produce the observed velocities in the absorption profiles. In this case, the predicted general relativistic precession periods are $\approx 20-30$ years. Thus, assuming that a complex of precessing gas rings is the correct geometry, the actual configuration should exist within this range.

The model line profiles are shown overlaid on the Fe II $5316 \AA$ data in Figure 3. In general, the precessing rings follow the evolution of the observed line profiles. The major exception is the model profile from 2015 April 11, which is weaker and shows a distinct absorption component near zero in Figure 1. There may be additional absorbing gas components that are responsible for the deviations from the ring profiles modeled here, which is plausible in the apparently dynamically active environment of this system. Thus, we have not attempted to accurately represent the entirety of absorbing gas during the 2015 April epoch. Furthermore, we do not attempt to model the zero velocity absorption component, which also seems to be present in the other line profiles at most epochs, since it appears to be fairly static. This component could be due to a stable outer gas ring with low eccentricity or a viewing angle that intersects a low line-of-sight velocity.

Figure 4 shows the absorption-weighted velocities of the observed Fe II $5316 \AA$ line profiles (pink diamonds) and the model line profiles (green line). The velocities are projected out to 2021 when the rings will have undergone a full precession period. The red error bars in Figure 4 are calculated by taking the standard deviation of the velocities that comprise $68 \%$ of the total absorption. The same calculation is performed for the model line profiles and this is shown with the gray band surrounding the model $V_{\text {avg }}$ values. Thus the larger uncertainties simply represent broader, flatter profiles. With the exception of the 2015 line profiles, the observed $V_{\text {avg }}$ values and line widths are in good agreement. The model predicts that the absorption will switch back to redshifted velocities sometime in mid-2019. Continued monitoring of WD $1145+017$ over the next two years will allow the precessing ring model to be robustly tested.

\section{Discussion}

The eccentric and precessing disk model discussed above can account for the evolving velocity shifts observed in the circumstellar absorption profiles toward WD $1145+017$.


Figure 3. Fe II $5316 \AA$ line profiles in the observer rest frame (black) and the corresponding eccentric disk model profiles (red) are shown in the left-hand panels. The right-hand panels show the configuration of gas rings at each epoch. The time in years from the 2015 April 11 observation is given by $\Delta t$. The colors change from red for the innermost and least eccentric rings to green for the outermost and most eccentric rings. The solid gray line indicates the line of sight to Earth and the dotted charcoal lines show the apsidal directions for the inner and outermost rings. Note that the apsidal directions are misaligned for the rings, a condition that is necessary in the model to reproduce the $\approx 200 \mathrm{~km} \mathrm{~s}^{-1}$ line widths.

Although the model successfully accounts for the broad morphological changes of the line profiles, we now discuss possible alternatives and outstanding issues with the favored scenario. 


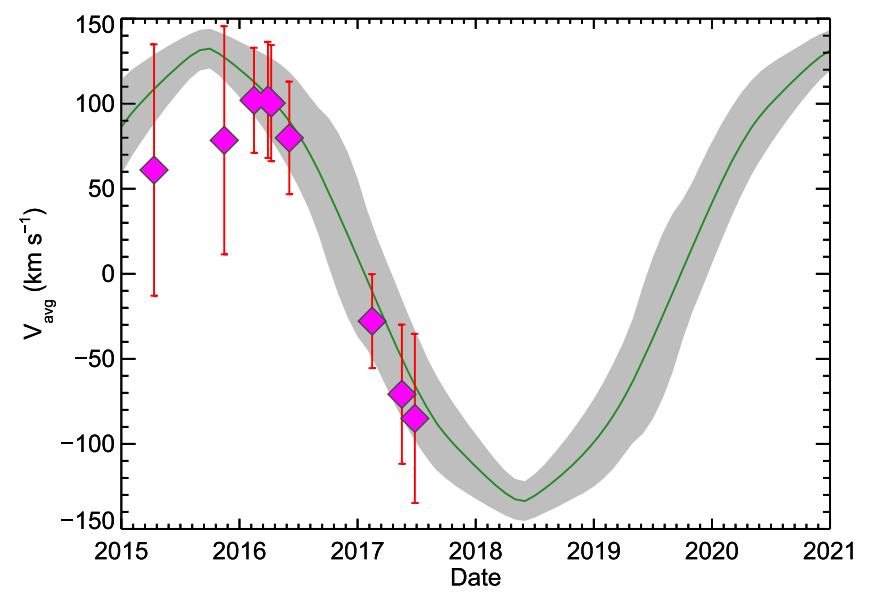

Figure 4. Absorption-weighted average velocities for the Fe II $5316 \AA$ line profiles (pink diamonds) and the corresponding model values from the eccentric disk profiles. The uncertainties (red lines and gray band) are calculated by taking the standard deviation of the velocities that comprise $68 \%$ of the measured absorption. The model velocities and line widths match the trend of the observed line profiles, although the 2015 lines are much broader than the model profiles. The model predicts that the profiles will switch back to being dominated by redshifted absorption sometime in mid-2019.

\subsection{Infalling/Accreting Material}

It has been suggested that the redshifted absorption velocities could be caused by infalling material being accreted onto the star (Xu et al. 2016). While this scenario is realistic for the redshifted absorption profiles, infalling material is inconsistent with the reversal into blueshifted absorption. The blueshifted profiles, if not due to the eccentric ring model presented here, require some sort of outflowing material, such as a disk or cometary wind. It is unlikely that we have observed the transition from accreting or infalling material into a wind or outflowing material, whereas the precessing rings explain the profile changes with no abrupt shifts in the circumstellar material geometry.

\subsection{High Eccentricity for Inner Gas Rings}

The eccentricities required in our model to produce the large observed velocities are in the range 0.25-0.30. Similar eccentricities have been proposed to explain the asymmetric $\mathrm{Ca}$ II infrared triplet emission line profiles observed in the Ton 345 system (Gänsicke et al. 2008; Melis et al. 2010), while a smaller eccentricity of $\approx 0.02$ was reported for SDSS $1228+1040$ (Gänsicke et al. 2006). These non-zero eccentricities have been questioned; however, and it has been suggested instead that the asymmetric Ca II line profiles, such as those observed for SDSS J122859.93 + 104032.9 (Manser et al. 2016), may be the result of non-uniform surface brightness in a circular gas disk (Metzger et al. 2012).

While non-axisymmetric disk surface brightness may be able to account for asymmetries in emission line profiles, it cannot yield the large maximum velocities and broad velocity range observed in the circumstellar absorption line profiles. Since the absorption arises along a narrow line of sight through the material, and thermal and turbulent line broadening cannot be greater than the local sound speed $\left(c_{s} \approx 1-2 \mathrm{~km} \mathrm{~s}^{-1}\right)$, large eccentricities are required to produce the observed line-of-sight velocities. As the eccentricity approaches zero, the line profiles become more symmetric about zero velocity and the maximum absorption velocities decrease. Although smaller eccentricities for smaller periastra can produce the large absorption velocities (due to the larger Keplerian orbital velocities at smaller orbital distances), these rings would precess far more quickly than is observed. Thus, the circumstellar lines observed and modeled here are consistent with the eccentric geometry suggested for the emitting gas disks in other white dwarf systems.

It has been suggested that rocky bodies orbiting near a white dwarf's Roche radius were initially in long period orbits at many astronomical units before being flung inward on highly eccentric orbits by massive planets (e.g., Bonsor et al. 2011; Veras et al. 2014). Radiation from the star can subsequently circularize the $10^{-4}-10 \mathrm{~cm}$ sized tidally disrupted debris in a small fraction of the white dwarf's cooling age (Veras et al. 2015). The eccentricity in the gas rings presented here could be leftover from this circularization process. Gurri et al. (2017) find that any large fragments around WD $1145+017$ are likely to be in nearly circular orbits if the parent body or bodies have masses greater than $10^{23} \mathrm{~g}$. However, there are no such eccentricity constraints for orbiting bodies with lower masses and this leaves significant phase space for eccentrically orbiting planetesimals and their debris, consistent with the gas in our model.

Finally, it is currently unclear what the circularization timescales are for eccentric rings of gas around white dwarfs. If the gas viscosity is sufficiently low, there will be little drag on the disk material and the circularization timescale may be long. Simulations of stellar tidal disruption around supermassive black holes show that the circularization timescale is strongly dependent on the eccentricity of the orbiting debris and the efficiency of energy dissipation in shocks generated by colliding material (e.g., Hayasaki et al. 2016). Smaller eccentricities, similar to the values in our model, imply lower relative velocities between intersecting gas streams and thus less energy dissipation in any resulting shocks, leading to longer circularization timescales. Detailed simulations of eccentric gas streams around white dwarfs are needed to provide constraints on this timescale for scenarios similar to the favored model.

\subsection{An External Perturber}

Previous to this study, the strongest evidence of a precessing gas disk has been observed in SDSS $1228+1040$. In that case, the data are consistent with a precession timescale of 24-30 years, and difficult to reconcile with an external, gravitational perturber such as a massive planet. Rather, the precession-like behavior of SDSS $1228+1040$ is consistent with the effects of general relativity (Manser et al. 2016). WD $1145+017$ is inferred to have asteroid- or planetesimal-sized solid bodies orbiting at $\approx 90 R_{*}$, and thus it is possible that more massive objects, i.e., planets, are orbiting undetected at large distances. As such, it is not implausible that an external perturber could be influencing secular variations in the disk.

The disk precession timescale due to external perturbing bodies can be estimated using dynamical simulations (Mustill $\&$ Wyatt 2009). Assuming orbital semimajor axes of $100 R_{*}$, $200 R_{*}$, and $2000 R_{*}$ and a perturbing mass of $1 M_{\mathrm{Jup}}$, the resulting precession periods are $\approx 6,45$, and 45,000 years. Assuming a smaller mass for the perturbing planet results in longer timescales. While the smallest precession period of $\approx 6$ years for a Jupiter-mass perturber at $100 R_{*}$ is similar to the observed evolution timescales of the line profiles, there is no evidence for such a massive companion at such small orbital 
distances. Furthermore, the Hill sphere of a Jupiter-mass planet at $100 R_{*}$ is $\approx 10 R_{*}$, which would likely disrupt the orbiting material near $90 R_{*}$. Thus the line profile changes are almost certainly not due to a massive perturbing body.

\section{Conclusions}

We have presented observations that reveal the complete velocity reversal of the circumstellar line profiles toward WD $1145+017$ over the course of $\approx 2$ years. The arguments from Section 4 suggest that eccentric rings precessing due to general relativistic effects are a reasonable explanation for the circumstellar profile variability. The system is highly dynamic, however, so it would not be surprising if the circumstellar profiles continue to evolve. Additional rings with varying eccentricity can be added to model any new features that arise in the future. Continued monitoring of WD $1145+017$ will aid in understanding the geometry of the innermost circumstellar gas explored here and provide constraints on white dwarf disk accretion models (e.g., Rafikov 2011; Metzger et al. 2012; Kenyon \& Bromley 2017a). If the eccentricity in the gas rings modeled here is intrinsic (rather than perturbative), their origin may be collisions within eccentric rings of solids, which is a long-expected but as-yet unconfirmed outcome of the tidally disrupted asteroid model for polluted white dwarf systems.

The authors acknowledge informative exchanges with D. Veras and N. C. Stone. A portion of the data presented herein was obtained at the W.M. Keck Observatory from telescope time allocated to the National Aeronautics and Space Administration through the agency's scientific partnership with the California Institute of Technology and the University of California. The Observatory was made possible by the generous financial support of the W.M. Keck Foundation. The authors wish to recognize and acknowledge the very significant cultural role and reverence that the summit of Maunakea has always had within the indigenous Hawaiian community. We are most fortunate to have the opportunity to conduct observations from this mountain. P.W.C. acknowledges support from the National Science Foundation through Astronomy and Astrophysics Research Grant AST-1313268 (PI: S.R.). S.B. and S.R. acknowledge the National Science Foundations support of the Keck Northeast Astronomy Consortiums REU program through grant AST-1005024. S.G.P. acknowledges the support of the Leverhulme Trust. The research leading to these results has received funding from the European
Research Council under the European Union's Seventh Framework Programme (FP/2007-2013)/ERC Grant Agreement No. 320964 (WDTracer). This work has made use of data from ESO proposal ID 598.C-0695. This work has made use of NASA's Astrophysics Data System.

\section{ORCID iDs}

Seth Redfield (iD https://orcid.org/0000-0003-3786-3486

\section{References}

Bonsor, A., Farihi, J., Wyatt, M. C., \& van Lieshout, R. 2017, MNRAS, 468, 154

Bonsor, A., Mustill, A. J., \& Wyatt, M. C. 2011, MNRAS, 414, 930

Croll, B., Dalba, P. A., Vanderburg, A., et al. 2017, ApJ, 836, 82

Farihi, J. 2016, NAR, 71, 9

Farihi, J., Gänsicke, B. T., \& Koester, D. 2013, Sci, 342, 218

Farihi, J., Parsons, S. G., \& Gänsicke, B. T. 2017, NatAs, 1, 32

Gänsicke, B. T., Aungwerojwit, A., Marsh, T. R., et al. 2016, ApJL, 818, L7

Gänsicke, B. T., Koester, D., Farihi, J., et al. 2012, MNRAS, 424, 333

Gänsicke, B. T., Koester, D., Marsh, T. R., Rebassa-Mansergas, A., \& Southworth, J. 2008, MNRAS, 391, 103

Gänsicke, B. T., Marsh, T. R., Southworth, J., \& Rebassa-Mansergas, A. 2006, Sci, 314, 1908

Gianninas, A., Strickland, B. D., Kilic, M., \& Bergeron, P. 2013, ApJ, 766, 3

Gurri, P., Veras, D., \& Gänsicke, B. T. 2017, MNRAS, 464, 321

Hayasaki, K., Stone, N., \& Loeb, A. 2016, MNRAS, 461, 3760

Jura, M., \& Young, E. D. 2014, AREPS, 42, 45

Kenyon, S. J., \& Bromley, B. 2017a, ApJ, 850, 50

Kenyon, S. J., \& Bromley, B. C. 2017b, ApJ, 844, 116

Koester, D., Gänsicke, B. T., \& Farihi, J. 2014a, A\&A, 566, A34

Koester, S., Gänsicke, B. T., \& Farihi, J. 2014b, A\&A, 566, 34

Manser, C. J., Gänsicke, B. T., Koester, D., Marsh, T. R., \& Southworth, J. 2016, MNRAS, 462, 1461

Melis, C., Jura, M., Albert, L., Klein, B., \& Zuckerman, B. 2010, ApJ, 722, 1078

Metzger, B. D., Rafikov, R. R., \& Bochkarev, K. V. 2012, MNRAS, 423, 505

Mustill, A. J., \& Wyatt, M. C. 2009, MNRAS, 399, 1403

Raddi, R., Gänsicke, B. T., Koester, D., et al. 2015, MNRAS, 450, 2083

Rafikov, R. R. 2011, MNRAS, 416, L55

Rappaport, S., Gary, B. L., Kaye, T., et al. 2016, MNRAS, 458, 3904

Redfield, S., Farihi, J., Cauley, P. W., et al. 2017, ApJ, 839, 42

Vanderburg, A., Johnson, J. A., Rappaport, S., et al. 2015, Natur, 526, 546

Veras, D., Leinhardt, Z. M., Bonsor, A., \& Gänsicke, B. T. 2014, MNRAS, 445,2244

Veras, D., Leinhardt, Z. M., Eggl, S., \& Gänsicke, B. T. 2015, MNRAS, 451,3453

Vernet, J., Dekker, H., D’Odorico, S., et al. 2011, A\&A, 536, A105

Vogt, S. S., Allen, S. L., Bigelow, B. C., et al. 1994, Proc. SPIE, 2198, 362

Xu, S., Jura, M., Dufour, P., \& Zuckerman, B. 2016, ApJL, 816, L22

Xu, S., Jura, M., Klein, B., Koester, D., \& Zuckerman, B. 2013, ApJ, 766, 132

Zuckerman, B., Melis, C., Klein, B., Koester, D., \& Jura, M. 2010, ApJ, 722,725 